\title{
X-RAY DIFFRACTION PATTERNS OF METAL AUROCYANIDES
}

\author{
Walter S. Selig \\ Gordon S. Smith \\ Kerstin K. Harding \\ and \\ Leslie 3. Summers
}

June 1989

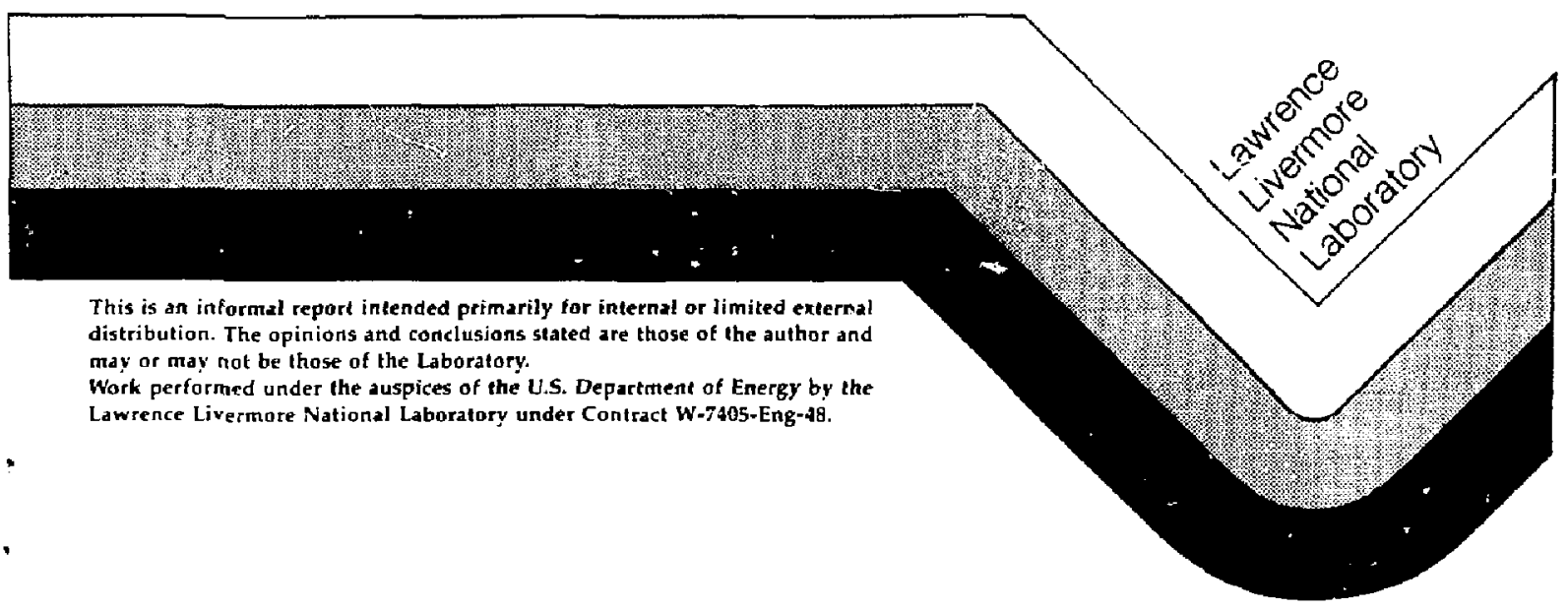




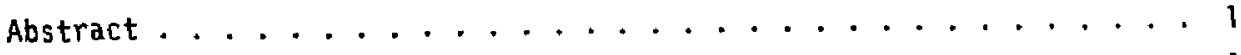

Introduction . . . . . . . . . . . . . . . . . . . . . . . . . . . 1

Experimental . . . . . . . . . . . . . . . . . . . . . . . . . . . . 1

Results and Discussion . . . . . . . . . . . . . . . . . . . . . . . . 3

Acknowledgment . . . . . . . . . . . . . . . . . . . . . . . . . . . 4

References . . . . . . . . . . . . . . . . . . . . . . . . . . . . . 4

\section{DISCLAIMER}

This report was prepared as an account of work sponsored by an agency of the United States Government. Neither the United States Government nor any agency thereof, nor any of their employees, makes any warranty, express or implied, or assumes any legal liability or responsibility for the accuracy, completeness, or uselulness of any information, apparatus, product, or process disclosed, or represents that its use would not infringe privalely ownet rights. Refer. ence herein to any specific commercial product, process, or service by trade name, trademark, manufacturer, or otherwise does not necessarily constitute or imply its endorsement. recons. mendation, or favoring by the United States Government of any agercy thereor. The views and opinions of authors expressed herein do not necessarily state or reflect those of the Uniled Stales Government of any agenc: :hetcol. 


\section{X-Ray Diffraction Patterns of Metal Aurocyanides}

Walter S. Selig, Gordon S. Smith, Kerstin K. Harding, and Leslie J. Summers Lawrence Livermore National Laboratory. University of California. P. O. Box 808, Livermore, CA 94550

\section{ABSTRACT}

Aurocyanides of the following metal cations have been prepared: $\mathrm{Ag}$, $\mathrm{Hg}(I I), \mathrm{Ga}, \mathrm{Fe}(I I I), \mathrm{Tl}(I), \mathrm{Bi}, \mathrm{Pb}, \mathrm{Mn}(I I), \mathrm{Ni}, \mathrm{Zn}, \mathrm{Cu}(\mathrm{II}), \mathrm{Cd}, \mathrm{In}$, and $C o(I I)$. Most of the aurocyanides are of the type $M\left[A u(C N)_{2}\right]_{x}$ where $M$ is the metal cation and $x$ its valence. However, under some conditions mixed aurocyanides containing $K$ may be formed, such as $K C O\left[A u(C N)_{2}\right]_{3}$. Only $\mathrm{Ag}$ and $\mathrm{Hg}$ (II) form aurocyanides which are sufficiently insoluble for the potentiometric determination of the aurocyanide anion. The diffraction patterns of the various aurocyanides are reported.

\section{INTRODUCTION}

We have previously reported new anaiytlcal methods for the determination of aurocyanide ion, $A u(C N)_{2}^{-}$, by potentiometric titration with silver ions (1) or with mercuric ions (2), according to the equation

$$
\left.\mathrm{M}^{\mathrm{X}+}+\mathrm{Au(CN)}\right)_{2}^{-} \longrightarrow \mathrm{M}\left[\mathrm{Au}(\mathrm{CN})_{2}\right]^{x}
$$

where $M$ is the metal cation and $x$ its valence.

Very little information is available on metal aurocyanides $(3,4)$. We have therefore prepared several metal aurocyanides and report their $x$-ray diffraction patterns here.

\section{EXPERIMENTAL}

Potassium aurocyanide, from Engelhard, had a nominal gold content of $68 \%$. The metal salts, with the exception of mercuric acetate, were nitrates. Their 
nominal degree of hydration is shown in Table 1 . Unmodified aqueous solutions of the materials were prepared at a concentration of $0.25 \mathrm{M}$. The solutior of mercuric acetate was acidified to $\mathrm{pH} 1.25$ with $1 \mathrm{~N}$ nitric acid, and bismuth nitrate was prepared in $1 \mathrm{M}$ nitric acid.

The metal aurocyanides were prepared by adding a $100 \%$ excess of the metal salt solution to $10 \mathrm{~mL}$ of $0.25 \mathrm{H}$ aurocyanide solution, heating on a hot-plate to near boiling, and cooling to ambient temperature. The resulting precipitates were filtered on No. 541 whatman paper, washed thoroughly with deionized water, and air-dried at ambient temperature for at least 48 hrs.

$X$-ray diffraction patterns were obtained with CuKa radiation, $\lambda=1.54050 \AA$.

Table 1. Nominal degree of hydration of metal salts used

Cation Number of water moleculesa

\begin{tabular}{ll}
\hline $\mathrm{Mn}$ & 51 soiution \\
$\mathrm{Fe}(\mathrm{III})$ & 9 \\
$\mathrm{Co}(\mathrm{II})$ & 6 \\
$\mathrm{Ni}$ & 6 \\
$\mathrm{Cu}$ & 2.5 \\
$\mathrm{In}$ & 6 \\
$\mathrm{Ga}$ & 9 \\
$\mathrm{Rh}$ & 3 \\
$\mathrm{Pd}(\mathrm{II})$ & 2 \\
$\mathrm{Ag}$ & 0 \\
$\mathrm{Cd}$ & 4 \\
$\mathrm{In}$ & 5 \\
$\mathrm{Hg}(I I)$ & 0 \\
$\mathrm{TI}(\mathrm{I})$ & 0 \\
$\mathrm{~Pb}$ & 0 \\
$\mathrm{Bi}$ & 5 \\
\end{tabular}

according to supplier 
Titrimetric methods for the determination of gold in plating solutions and in potassium aurocyanide usually require oxidation of gold from the monovalent to the trivalent state. After this conversion, various redox methods (5) are available for the determination. Another technique, controlled-potential coulometry, has been used at LLNL for many years as the primary assay technique for plating solutions $(6,7)$. This technique also requires that gold be in the trivalent state. This conversion requires treatment with aqua regia. Monovalent gold as aurocyanide can be determined wi thout such treatment, using silver (1) or mercuric (2) ions as titrants, according to equation (1). The methods can be applied on the microscale, with $10^{-3} \mathrm{M}$ solutions, using a $0.01 \mathrm{M}$ titrant.

According to an ancient reference (8) potassium aurocyanide yields precipitates with $\mathrm{Fe}(\mathrm{II}), \mathrm{Cu}(\mathrm{II}), \mathrm{Zn}, \mathrm{Ag}, \mathrm{Hg}(\mathrm{II})$, and $\mathrm{Pb}$ lons. This prompted us to attempt using these cations as possible titrants for aurocyanide. We found that on $\mathrm{Y} \mathrm{Ag}$ and $\mathrm{Hg}$ (II) ions yielded precipitates in very dilute solutions. The other cations, as well as those ifsted in Table 1, yielded precipitates only in more concentrated solutions, as outlined in the experimental section. This indicates that except for silver and mercuric aurocyanide, the aurocyanides have appreciable solubility in water. $F e$ (II) did not yieid a precipitate even from $0.25 \mathrm{M}$ solution.

To the best of our knowledge only the aurocyanides of Ag (4), TI(I) (9), and $C o(I I)(10)$ have been reported in the literature. Only for the cobalt compound have $X$-ray powder patterns been reported (patterns nos. $31-420$ and $37-565$ in reference 11$)$. This work was done by Eisenmann who reported the compound $\mathrm{KCO}\left[\mathrm{Au}(\mathrm{CH})_{2}\right]_{3}$, formed by using an excess of potassium aurocyanide, according to the equation

$$
3 \mathrm{KAu}[\mathrm{CN}]_{2}+\mathrm{Co}\left(\mathrm{NO}_{3}\right)_{2} \longrightarrow \mathrm{KCo}\left[\mathrm{Au}(\mathrm{CN})_{2}\right]_{3}+2 \mathrm{KNO}_{3}
$$

We confirmed the formation of this compound and have listed its diffraction pattern in Table 15. The pattern is similar, though not identical, with that presented in reference 11 . The presence of potassium was confirmed by $X$-ray fluorescence spectroscopy ( $>1 \%$ found vs a theoretical 4.6\%). Eisenmann found that recrystallization of $\mathrm{KCO}\left[\mathrm{Au}(\mathrm{CN})_{2}\right]_{3}$ from water 
resulted in a compound yielding a different diffraction pattern, which contained no potassium and had a Au:Co ratio of $\left.2: 1, \mathrm{COAu}_{\mathrm{CN}}\right]_{2}$. The diffraction pattern of the decahydrate of this compound is listed as no. 31-420 in reference 11 . We obtained a similar, though not identical, diffraction pattern by using an excess of potassium aurocyanide in the formation of this compound as shown in Table 16. This compound, as well as the others listed in the tables, were forned according to equation (1). Further work needs to be done to deterintne whether equation (2) applies in every case where an excess of the heavy metal nitrate is used.

Rh(III) yielded an anorphous material with potassium aurocyanide, while Pd(II) produced a weakly crystalline material.

We have previously found that the powder pattern of silver aurocyanide is isomorphous with that of AuCN (12). The diffraction patterns of the aurocyanides of $\mathrm{Bi}, \mathrm{Pb}, \mathrm{Ni}, \mathrm{Cu}, \mathrm{Cd}$, and $\mathrm{Co}$ (Tables $7,8,10,12,13$, and 16) also appear to be isomorphous.

\section{ACKNOWLEDGNENT}

The writers thank Darrell K. Pugh for the $x$-ray fluorescence spectroscopy.

\section{REFERENCES}

1. W. S. Selig. Plating Surf. Fin. $72(11), 70(1985)$.

2. W. S. Selig, Microchem. J. 38, 332 (1988).

3. A. G. Sharpe. "The Chemistry of Cyano Complexes of the Transition Metals, Academic Pres5, New York, p. 275-277 (1976).

4. A. Bodensiek, Diss. Hannover T. H., 1925; through "Gmel in Handbuch der Anorganischen Chemie," System No. 62: Gold, 8th ed., Verlag Chemie, Weinheim-Germany, p. 767 (1954).

5. D. G. Foulke (Ed.), "Electroplaters" Process Control Handbook," revised ed., Huntington, New York, p. 195-197 (1975).

6. J. E. Harrar and M. C. Waggoner, Plating Surf, Fin. 68, 41 (1981).

7. J. E. Harrar and R. B. Stephens, 3. Electroanal. Chem. 3, 112 (1962). 
8. C. F. O. Glassford and J. Napier, Phil Mag. [3] 25, 56 (1844); through Gmelin, ref. 4 (above), p. 744.

9. H. Stammreich, B. H. Chadwick, and S. G. Frankiss, J. Mol. Structure I, 191 (1967).

10. E. T. Eisenmann, I. Electrochem. Soc. 124, 1957 (1977).

11. "Powder Diffraction File," JCPOC International Centre for Diffraction Data, 1601 Park Lane, Swarthmore, PA 19081.

12. W. S. Selig, "Final Analysis" in "Readers Speak", Plating Surf. Fin. 76(5), 10 (May 1988).

Table 2. X-ray diffraction pattern of $\mathrm{Ag}\left[\mathrm{Au}(\mathrm{CN})_{2}\right]$

\begin{tabular}{lrrr}
\hline$d(A)$ & height & $d(A)$ & height \\
\hline 5.12 & 64 & 1.615 & 8 \\
2.953 & 100 & 1.600 & 4 \\
2.570 & 61 & 1.511 & 3 \\
1.712 & 22 & 1.481 & 14 \\
1.705 & 17 & 1.105 & 1 \\
\hline
\end{tabular}


Table 3. X-ray diffraction pattern of $\mathrm{Hg}\left[\mathrm{Au}(\mathrm{CN})_{2}\right]_{2}$

\begin{tabular}{|c|c|c|c|}
\hline$d(\AA)$ & height & $d(\AA)$ & height \\
\hline 8.91 & 17 & 1.795 & 21 \\
\hline 7.44 & $<1$ & 1.677 & 11 \\
\hline 4.73 & 11 & 1.648 & 10 \\
\hline 4.47 & 78 & 1.627 & 17 \\
\hline 4.18 & 72 & 1.600 & 23 \\
\hline 3.349 & 3 & 1.581 & 27 \\
\hline 3.253 & 8 & 1.557 & 28 \\
\hline 3.136 & 100 & 1.540 & 29 \\
\hline 2.992 & 67 & 1.528 & 18 \\
\hline 2.840 & 77 & 1.500 & 8 \\
\hline 2.525 & 24 & 1.494 & 14 \\
\hline 2,490 & 39 & 1.478 & 4 \\
\hline 2.368 & 19 & 1.462 & 6 \\
\hline 2.231 & 44 & 1.429 & 12 \\
\hline 2.117 & 50 & 1.429 & 10 \\
\hline 2.094 & 24 & 7.423 & 8 \\
\hline 2.025 & 4 & 1.398 & 15 \\
\hline 1.916 & 41 & 1.338 & 9 \\
\hline 1.859 & 3 & 1.317 & 9 \\
\hline 1.820 & 2 & 1.302 & 7 \\
\hline
\end{tabular}


Table 4. X-ray diffraction pattern of $\mathrm{Ga}\left[\mathrm{Au}(\mathrm{CN})_{2}\right]_{3}$

\begin{tabular}{rrrr}
\hline$d(A)$ & height & $d(A)$ & height \\
\hline 15.45 & 5 & 2.579 & 35 \\
10.27 & 51 & 2.395 & 2 \\
7.76 & 2 & 2.312 & 31 \\
5.15 & 94 & 2.063 & 4 \\
3.851 & 2 & 1.988 & 33 \\
3.434 & 17 & 2.802 & 10 \\
3.118 & 100 & 1.776 & 7 \\
2.988 & 64 & 1.720 & 17 \\
2.846 & 3 & 1.701 & 8 \\
2.671 & 49 & 1.595 & 3 \\
2.631 & 49 & 1.562 & 3 \\
\hline
\end{tabular}

Table 5. X-ray diffraction pattern of Fe[Au(CN) $\left.{ }_{2}\right]_{3}$

\begin{tabular}{crrr}
\hline$d(\xi)$ & height & $d(\AA)$ & Leight \\
\hline 10.22 & 52 & 2.067 & 7 \\
5.15 & 100 & 1.983 & 35 \\
2.440 & 22 & 1.792 & 8 \\
3.093 & 70 & 1.763 & 7 \\
2.961 & 47 & 1.723 & $2 i$ \\
2.653 & 43 & 1.691 & 8 \\
2.582 & 46 & 1.590 & 4 \\
2.301 & 29 & & \\
& & &
\end{tabular}


Table 6. X-ray diffraction pattern of $T I\left[A u(C N)_{2}\right]$

\begin{tabular}{|c|c|c|c|}
\hline$d(A)$ & height & $d(A)$ & height \\
\hline 7.51 & 3 & 2.235 & 14 \\
\hline 5.87 & 5 & 2.218 & 8 \\
\hline 5.76 & 7 & 2.189 & 10 \\
\hline 4.87 & 3 & 2.151 & 37 \\
\hline 4.59 & 15 & 2.104 & 100 \\
\hline 4.49 & 27 & 2.080 & 9 \\
\hline 4.41 & 25 & 1.999 & 2 \\
\hline 4.10 & 10 & 1.960 & 13 \\
\hline 3.903 & 9 & 1.958 & 6 \\
\hline 3.792 & 12 & 1.936 & 10 \\
\hline 3.717 & 38 & 1.919 & 11 \\
\hline 3.587 & 3 & 1.891 & 12 \\
\hline 3.424 & 2 & 1.879 & 5 \\
\hline 3.206 & 97 & 1.865 & 6 \\
\hline 3.140 & 71 & 1.838 & 1 \\
\hline 3.056 & 11 & 1.796 & 4 \\
\hline 2.989 & 83 & 1.771 & 11 \\
\hline 2.919 & 5 & 1.755 & 5 \\
\hline 2.879 & 6 & 1.727 & 10 \\
\hline 2.798 & 21 & 1.699 & 4 \\
\hline 2.764 & 4 & 1.675 & 22 \\
\hline 2.696 & 8 & 1.659 & 3 \\
\hline 2.629 & 2 & 1.643 & 7 \\
\hline 2.590 & 26 & 1.635 & 10 \\
\hline 2.531 & 22 & 1.616 & 5 \\
\hline 2.383 & 4 & 1.607 & 14 \\
\hline 2.354 & 17 & 1.583 & 18 \\
\hline 2.306 & 9 & 1.548 & 12 \\
\hline 2.283 & 12 & & \\
\hline
\end{tabular}


Table 7. X-ray diffraction pattern of $B i\left[A U(C N)_{2}\right]_{3}$

\begin{tabular}{|c|c|c|c|}
\hline$d(A)$ & height & $d(A)$ & helght \\
\hline 11.86 & 2 & 2.442 & 70 \\
\hline 9.13 & 40 & 2.283 & 45 \\
\hline 5.97 & 16 & 2.250 & 16 \\
\hline 5.49 & 2 & 2.212 & 2 \\
\hline 4.88 & 56 & 2.126 & 20 \\
\hline 4.56 & 100 & 2.096 & 10 \\
\hline 3.965 & 5 & 2.049 & 2 \\
\hline 3.870 & 9 & 2.006 & 2 \\
\hline 3.509 & 7 & 1.828 & $?$ \\
\hline 3.243 & 22 & 1.825 & 5 \\
\hline 3.136 & 26 & 1.792 & 41 \\
\hline 3.093 & 5 & 1.775 & 15 \\
\hline 3.044 & 16 & 1.741 & 23 \\
\hline 3.004 & 3 & 1.670 & 8 \\
\hline 2.930 & $3 i j$ & 1.642 & 4 \\
\hline 2.758 & 18 & 1.629 & 6 \\
\hline 2.731 & 11 & 1.601 & 7 \\
\hline 2.693 & 33 & 1.581 & 7 \\
\hline 2.635 & 12 & 1.568 & 15 \\
\hline
\end{tabular}


Table 8. X-ray diffraction pattern of $\mathrm{Pb}\left[\mathrm{Au}(\mathrm{CN})_{2}\right]_{2}$

\begin{tabular}{|c|c|c|c|}
\hline$d(\AA)$ & height & $d(A)$ & height \\
\hline 9.73 & 12 & 2.229 & 36 \\
\hline 6.54 & 7 & 2.198 & 40 \\
\hline 6.08 & 1 & 2.167 & 13 \\
\hline 5.44 & 7 & 2.142 & 2 \\
\hline 5.15 & 1 & 2.087 & 5 \\
\hline 4.88 & 100 & 2.044 & 1 \\
\hline 4.75 & 22 & 1.999 & 12 \\
\hline 4.27 & 13 & 1.983 & 9 \\
\hline 3.924 & 4 & 1.967 & 6 \\
\hline 3.804 & 1 & 1.924 & 6 \\
\hline 3.699 & 7 & 7.910 & 5 \\
\hline 3.440 & 20 & 1.881 & 3 \\
\hline 3.408 & 21 & 1.852 & 12 \\
\hline 3.329 & 5 & 1.817 & 5 \\
\hline 3.290 & 16 & 1.814 & 5 \\
\hline 3.261 & 10 & 1.784 & 2 \\
\hline 3.149 & 25 & 1.758 & 3 \\
\hline 3.113 & 44 & 1.732 & 14 \\
\hline 3.047 & 17 & 1.705 & 7 \\
\hline 2.912 & 80 & 1.686 & 12 \\
\hline 2.839 & 51 & 1.667 & 3 \\
\hline 2.811 & $i 4$ & 1.651 & 17 \\
\hline 2.732 & 5 & 1.635 & 33 \\
\hline 2.706 & 8 & 1.625 & 13 \\
\hline 2.611 & 6 & 1.612 & 5 \\
\hline 2.591 & 8 & 1.603 & וו \\
\hline 2.451 & 62 & 1.588 & 14 \\
\hline 2.371 & 4 & 1.567 & 7 \\
\hline 2.319 & 26 & & \\
\hline
\end{tabular}


Table 9. X-ray diffraction pattern of $M n\left[A u(C N)_{2}\right]_{2}$

\begin{tabular}{|c|c|c|c|}
\hline$d(A)$ & helght & $d(A)$ & height \\
\hline 15.41 & 13 & 2.242 & 6 \\
\hline 8.01 & 80 & 2.216 & 1 \\
\hline 7.72 & 5 & 2.159 & 16 \\
\hline 5.92 & 10 & 2.108 & 14 \\
\hline 5.16 & 26 & 2.005 & 34 \\
\hline 4.75 & 69 & 1.984 & 36 \\
\hline 4.01 & 59 & 1.957 & 8 \\
\hline 3.876 & 3 & 1.920 & 11 \\
\hline 3.424 & 7 & 1.898 & 4 \\
\hline 3.319 & 14 & 1.801 & 2 \\
\hline 3.149 & 45 & 1.789 & 2 \\
\hline 3.101 & 4 & 1.774 & 3 \\
\hline 3.036 & 17 & 1.726 & 5 \\
\hline 2.964 & 69 & 1.713 & 19 \\
\hline 2.829 & 3 & 1.675 & 14 \\
\hline 2.781 & 100 & 1.660 & 24 \\
\hline 2.699 & 6 & 1.611 & 6 \\
\hline 2.675 & 40 & 1.603 & 6 \\
\hline 2.617 & 10 & 1.590 & 9 \\
\hline 2.588 & 11 & 1.575 & 23 \\
\hline 2.435 & 15 & 1.548 & 8 \\
\hline 2.383 & 88 & & \\
\hline
\end{tabular}


Table 10. X-ray diffraction pattern of $\mathrm{Ni}\left[\mathrm{Au}(\mathrm{CN})_{2}\right]_{2}$

\begin{tabular}{cccc}
\hline$d(A)$ & height & $d(A)$ & height \\
\hline 10.13 & 3 & 2.559 & 16 \\
7.62 & 51 & 2.340 & 91 \\
5.87 & 9 & 2.231 & 7 \\
5.10 & 4 & 2.144 & 16 \\
4.67 & 66 & 2.047 & 14 \\
3.830 & 39 & 1.935 & 38 \\
3.403 & 7 & 1.907 & 10 \\
3.216 & 16 & 1.828 & 3 \\
3.110 & 43 & 1.752 & 2 \\
3.036 & 3 & 1.706 & 12 \\
2.949 & 68 & 1.684 & 11 \\
2.819 & 2 & 1.666 & 14 \\
2.754 & 100 & 1.641 & 1 \\
2.699 & 1 & 1.611 & 29 \\
& & 1.560 & 29 \\
& & & \\
\hline
\end{tabular}


Table 11. X-ray diffraction pattern of $Z n\left[A u(C N)_{2}\right]_{2}$

\begin{tabular}{rrrr}
\hline$d(A)$ & height & $d(A)$ & height \\
\hline 6.78 & 41 & 2.106 & 19 \\
4.98 & 5 & 2.014 & 47 \\
4.11 & 100 & 1.952 & 3 \\
4.12 & 15 & 1.883 & 27 \\
3.593 & 69 & 1.821 & 13 \\
3.257 & 12 & 1.773 & 4 \\
3.220 & 13 & 1.718 & 7 \\
2.976 & 64 & 1.672 & 37 \\
2.731 & 61 & 1.631 & 3 \\
2.433 & 65 & 1.595 & 34 \\
2.294 & 82 & 1.552 & 32 \\
2.199 & 1 & & - \\
\hline
\end{tabular}

Table 12. X-ray diffraction pattern of $\mathrm{Cu}\left[\mathrm{Au}(\mathrm{CN})_{2}\right]_{2}$

\begin{tabular}{rrrr}
\hline$d(\AA)$ & height & $d(\AA)$ & hejght \\
\hline 10.12 & 93 & 2.319 & 15 \\
6.01 & 6 & 2.283 & 13 \\
5.10 & 100 & 2.261 & 9 \\
3.409 & 28 & 2.142 & 3 \\
3.335 & 10 & 2.047 & 6 \\
3.162 & 40 & 1.991 & 13 \\
3.068 & 30 & 1.967 & 15 \\
3.019 & 66 & 1.954 & 15 \\
2.938 & 22 & 1.820 & 6 \\
2.897 & 17 & 1.787 & 6 \\
2.687 & 20 & 1.708 & 20 \\
2.628 & 18 & 1.646 & 3 \\
2.602 & 21 & 1.584 & 3 \\
2.559 & 40 & 1.562 & 1 \\
\hline
\end{tabular}


Tabie 13. X-ray diffraction pattern of $\mathrm{Cd}\left[\mathrm{Au}(\mathrm{CN})_{2}\right]_{2}$

\begin{tabular}{rrrr}
\hline$d(\AA)$ & height & $d(\AA)$ & height \\
\hline .09 & 72 & 2.132 & 9 \\
5.89 & 1 & 2.047 & 53 \\
4.76 & 18 & 2.008 & 11 \\
4.07 & 100 & 1.963 & 1 \\
3.409 & 1 & 1.935 & 1 \\
3.358 & 3 & 1.919 & 5 \\
3.149 & 13 & $1.73 ?$ & 6 \\
2.957 & 17 & 1.712 & 3 \\
2.777 & 16 & 1.686 & 20 \\
2.725 & 35 & 1.675 & 2 \\
2.477 & 14 & 1.640 & 4 \\
2.397 & 25 & 1.612 & 3 \\
2.240 & 1 & 1.602 & 3 \\
2.159 & 6 & 1.580 & 12 \\
\hline
\end{tabular}


Table 14. X-ray diffraction pattern of $\operatorname{In}\left[A u(C N)_{2}\right]_{3}$

\begin{tabular}{|c|c|c|c|}
\hline$d(A)$ & helght & $d(A)$ & height \\
\hline 7.97 & 33 & 1.657 & 16 \\
\hline 5.70 & 1 & 1.647 & 43 \\
\hline 4.66 & 78 & 1.624 & 1.3 \\
\hline 4.01 & 34 & 1.607 & 3 \\
\hline 3.309 & 5 & 1.559 & 12 \\
\hline 3.060 & 40 & 1.548 & 8 \\
\hline 2.868 & 74 & 1.533 & 26 \\
\hline 2.699 & 61 & 1.447 & 6 \\
\hline 2.674 & 13 & 1.437 & 10 \\
\hline 2.556 & 1 & 1.411 & 11 \\
\hline 2.428 & 21 & 1.404 & 11 \\
\hline 2.335 & 100 & 1.370 & 6 \\
\hline 2.169 & 2 & 1.352 & 14 \\
\hline 2.094 & 29 & 1.341 & 4 \\
\hline 2.083 & 21 & 1.300 & 5 \\
\hline 2.009 & 13 & 1.293 & 7 \\
\hline 1.959 & 23 & 1.280 & 14 \\
\hline 1.909 & 1 & 1.267 & 6 \\
\hline 1.862 & 11 & 1.239 & 4 \\
\hline 1.729 & 1 & 1.232 & 2 \\
\hline 1.686 & 18 & 1.215 & 14 \\
\hline
\end{tabular}


Table 15. X-ray diffraction pattern of $K C O\left[A u(C N)_{2}\right]_{3}$

\begin{tabular}{lrrr}
\hline$d(A)$ & height & $d(A)$ & height \\
\hline 7.751 & 100 & 2.147 & 11 \\
5.901 & 7 & 2.067 & 12 \\
4.706 & 43 & 1.951 & 46 \\
3.897 & 55 & 1.910 & 8 \\
3.408 & 4 & 1.852 & 4 \\
3.252 & 12 & 1.758 & 2 \\
3.123 & 27 & 1.707 & 13 \\
2.953 & 42 & 1.696 & 10 \\
2.761 & 61 & 1.667 & 10 \\
2.599 & 28 & 1.628 & 31 \\
2.391 & 14 & 1.605 & 5 \\
2.354 & 63 & 1.563 & 22 \\
2.235 & 5 & & \\
\hline
\end{tabular}

Table 16. X-ray diffraction pattern of Co[Au(CN) $\left.{ }_{2}\right]_{2}$ (or decahydrate)

\begin{tabular}{rrrr}
\hline$d(\AA)$ & $\begin{array}{c}d(\AA) \\
\text { height }\end{array}$ & height & \\
\hline 10.040 & 8 & 2.051 & 3 \\
5.093 & 15 & 1.996 & 10 \\
3.409 & 5 & 1.941 & 19 \\
3.184 & 55 & 1.800 & 30 \\
3.040 & 42 & 1.772 & 17 \\
2.972 & 100 & 1.699 & 23 \\
2.853 & 54 & 1.685 & 25 \\
2.706 & 18 & 1.663 & 8 \\
2.564 & 43 & 1.597 & 27 \\
2.328 & 12 & 1.578 & 9 \\
2.241 & 19 & &
\end{tabular}

